job-related satisfaction in addition to satisfaction with one's job itself. Although the measures suggested by Dr Kader would have been appropriate, some of their items overlapped with the Karasek Job Content Questionnaire (Karasek, 1979), and we were keen to avoid such duplication and overburdening respondents. As we were interested in the relationship between satisfaction with one's job and other indicators of job-related satisfaction such as feelings about pay, operational and policy contexts (which were and remain topical because of proposed changes to the Mental Health Act 1983) and feeling valued, it would have been inappropriate to use a multi-faceted job satisfaction scale as a dependent variable. All of the scales used in the survey are well known and have established reliability and validity.

The adjusted response rate of $49 \%$, although low in comparison with experimental studies, is very reasonable for social surveys of this type. We agree that it would have been helpful to know how non-respondents compared with respondents in terms of demographic and other details, but the methodology meant that was not achievable. Nevertheless, we do know that our sample was very similar, demographically and in terms of tenure, length of experience, approved social worker status, etc., to another recent study of mental health social workers (ADSS Cymru, 2005). Therefore we have no reason to believe that these data are not representative.

Finally, although it might have been interesting to present a stepwise regression model, we opted for an 'enter' model in the interests of brevity. Subsequent analyses have shown that a stepwise approach offers little added value.

Like Dr Kader, we hope that the results of our survey are an eye-opener for employers.

ADSS Cymru (2005) Social Workers in Wales: A Profession to Value. Cardiff: ADSS Cymru.

Karasek, R. (1979) Job demands, job decision latitude and mental strain. Implications for job redesign. Administrative Quarterly, 24, 285-308.

S. Evans Social Work and Social Care Section, Health Services Research Department, Institute of Psychiatry, and Social Care Workforce Research Unit, King's College London, London, UK. Email: S.Evans@iop.kcl.ac.uk

P. Huxley Health Services Research

Department, Institute of Psychiatry, and Social Care Workforce Research Unit, King's College London,

London, UK

doi: 10.II92/bjp.I89.I.84b

\section{Psychological factors in bipolar disorder}

Jones et al (2005) have focused on the important although relatively neglected area of psychosocial aspects/intervention in bipolar affective disorder. Although there are several previous reports on the subject by the same group, this study has a better design and a much larger sample size. However, some central issues remain unresolved.

The authors were unable to find dysfunctional beliefs specific to bipolar disorder. Cognitive therapy as practised in depressive or panic disorders attempts to correct characteristic dysfunctional beliefs (Beck \& Rush, 2000). In the absence of a specific pattern of dysfunctional beliefs, devising effective and specific cognitive strategies to treat bipolar disorder may be difficult. This is illustrated by the pilot study of cognitive therapy in bipolar disorders by the same group (Scott et al, 2001) in which relatively non-specific strategies such as self-management of symptoms, dealing with non-adherence, anti-relapse techniques, etc. were employed. The lack of precise techniques could also have resulted in the differential efficacy of cognitive therapy, with effects mainly on depressive, rather than manic symptoms.

In the current study Jones et al used a 24-item sub-scale version of the Dysfunctional Attitude Scale, whereas in earlier studies (Scott et al, 2000; Scott \& Pope, 2003) a 40 -item scale was used. It is not clear whether the use of different versions of this scale contributed to the ambiguous nature of the dysfunctional beliefs found in bipolar disorder, especially since the two different versions appear to have different sub-scales. Finally, although some potential confounding variables, such as current mental state, were controlled for, others, such as duration of illness, severity, chronicity and possible effects of pharmacophrophylaxis, were not. Cognitive style may vary according to these factors (Scott $\&$ Pope, 2003) making it necessary to control for them.

It is possible that these concerns will be addressed by future research. This study paves the way for examination of psychosocial factors in bipolar disorder.

Beck, A. T. \& Rush, A. J. (2000) Cognitive therapy. In Comprehensive Textbook of Psychiatry (Vol. 7) (eds H. I. Kaplan \& B. J. Saddock), pp. 2167-2178. Baltimore, MD: Williams \& Wilkins.
Jones, L., Scott, J., Haque, S., et al (2005) Cognitive style in bipolar disorder. British Journal of Psychiatry, $\mathbf{1 8 7}$ 43I-437.

Scott, J. \& Pope, M. (2003) Cognitive style in individuals with bipolar disorders. Psychological Medicine, 33, 1082-1088.

Scott, J., Stanton, B., Garland, A., et al (2000) Cognitive vulnerability in bipolar disorders. Psychological Medicine, 30, 467-472.

Scott, J., Garland, A. \& Moorhead, S. (200I) A pilot study of cognitive therapy in bipolar disorders. Psychological Medicine, 3I, 459-467.

P. Biswas Department of Psychiatry, Postgraduate Institute of Medical Education and Research, Chandigarh 160012, India.

Email: ps_bs69@yahoo.com

S. Chakrabarti Department of Psychiatry, Postgraduate Institute of Medical Education and Research, Chandigarh, India

doi: 10.II92/bjp.I89.I.85

Authors' reply: We are pleased that Biswas \& Chakrabarti highlight the strengths of our study design and large sample size, and consider our work a significant contribution to understanding psychological factors in bipolar disorder. We agree that it is important to consider potential confounders and therefore examined the effects of differences in illness duration and severity. Although there were some differences between our two patient groups on measures of illness severity and a small number of modest correlations between illness severity and cognitive style, covarying for these measures had no effect on our finding that those with bipolar disorder have fragile self-esteem and dysfunctional beliefs similar to those of people with unipolar disorder. We have not been able to examine the possible effects of pharmacoprophylaxis on cognitive style, but agree that this could be a target for future research.

We do not think we would have found differences in cognitive style between participants with bipolar and unipolar disorder if we had used a longer version of the Dysfunctional Attitudes Scale (DAS). The 24-item version used in our study was factor-analytically derived from the longer version and has improved robustness (Power et al, 1994). The 'need for achievement' and 'dependency' sub-scales of the 24-item DAS comprise items from the 'perfectionism' and 'need for approval' subscales of the 40-item DAS respectively.

We hope that future studies of cognitive style in people with mood disorder will build on the strengths of our study by using 